\title{
White matter tract covariance patterns predict age-declining cognitive abilities
}

\author{
Yunglin Gazes a,*, F. DuBois Bowman ${ }^{\text {b }}$, Qolamreza R. Razlighi ${ }^{\text {a }}$, Deirdre O'Shea a , \\ Yaakov Stern ${ }^{a}$, Christian Habeck ${ }^{\mathrm{a}}$ \\ a Cognitive Neuroscience Division, Department of Neurology, Columbia University Medical Center, New York, NY, USA \\ ${ }^{\mathrm{b}}$ Department of Biostatistics, Mailman School of Public Health, Columbia University, New York, NY, USA
}

\section{A R T I C L E I N F O}

\section{Article history:}

Received 5 June 2015

Accepted 7 October 2015

Available online 19 October 2015

\section{Keywords:}

Diffusion tensor imaging

Multivariate statistics

Principal component analysis

Mediation

Cognition

\begin{abstract}
A B S T R A C T
Previous studies investigating the relationship of white matter (WM) integrity to cognitive abilities and aging have either focused on a global measure or a few selected WM tracts. Ideally, contribution from all of the WM tracts should be evaluated at the same time. However, the high collinearity among WM tracts precludes systematic examination of WM tracts simultaneously without sacrificing statistical power due to stringent multiplecomparison corrections. Multivariate covariance techniques enable comprehensive simultaneous examination of all WM tracts without being penalized for high collinearity among observations.

Method: In this study, Scaled Subprofile Modeling (SSM) was applied to the mean integrity of 18 major WM tracts to extract covariance patterns that optimally predicted four cognitive abilities (perceptual speed, episodic memory, fluid reasoning, and vocabulary) in 346 participants across ages 20 to 79 years old. Using expression of the covariance patterns, age-independent effects of white matter integrity on cognition and the indirect effect of WM integrity on age-related differences in cognition were tested separately, but inferences from the indirect analyses were cautiously made given that cross-sectional data set was used in the analysis.

Results: A separate covariance pattern was identified that significantly predicted each cognitive ability after controlling for age except for vocabulary, but the age by WM covariance pattern interaction was not significant for any of the three abilities. Furthermore, each of the patterns mediated the effect of age on the respective cognitive ability. A distinct set of WM tracts was most influential in each of the three patterns. The WM covariance pattern accounting for fluid reasoning showed the most number of influential WM tracts whereas the episodic memory pattern showed the least number.

Conclusion: Specific patterns of WM tracts make significant contributions to the age-related differences in perceptual speed, episodic memory, and fluid reasoning but not vocabulary. Other measures of brain health will need to be explored to reveal the major influences on the vocabulary ability.
\end{abstract}

(c) 2015 Elsevier Inc. All rights reserved.

\section{Introduction}

Investigating the structural differences across age groups and how that relates to differences in cognition will contribute to further understanding of the aging process. After repeated replications, Salthouse (2005) and Salthouse et al. (2008) have shown that age-related differences in cognitive performance can be parsimoniously represented as four cognitive abilities: perceptual speed, working memory, fluid ability, and vocabulary. Under this framework, identifying the underlying differences in structural integrity for each cognitive ability provides a comprehensive examination of the set of white matter (WM) structures that contribute to the aging mechanism. In monkeys, WM damage was found to be the most powerful driving force in the mechanism of cognitive decline (Moss et al., 2007). Similarly, WM integrity measured with

\footnotetext{
* Corresponding author at: 630 W 168th Street, P\&S Box 16, New York, NY 10032, USA. E-mail address: YL2107@cumc.columbia.edu (Y. Gazes).
}

diffusion tensor imaging was also reported to show the strongest associations with age-related differences in cognition among other imaging modalities in humans (Hedden et al., 2014). Therefore, the main research question in the current study was whether variability in major WM tracts (1) exhibit age-independent effects on cognition and (2) mediate the age-related differences in these four cognitive abilities in healthy adults across the full age range of adulthood. We hypothesized that each of these abilities relies on a specific set of WM tracts that connects a network of brain regions involved in processing that ability, and thus, a different WM covariance pattern, consisting of a set of tracts that covary across people, would be extracted for each cognitive ability that both exhibits age-independent effects on cognition and mediates the age-related differences in each cognitive ability.

Intactness of WM microstructure can be approximated by diffusion tensor imaging (DTI), which is sensitive to signal changes due to water diffusion in the brain. DTI indirectly measures the integrity as well as the direction of gross axonal connections by fitting an ellipsoid 
at each voxel characterized by three perpendicular axes. Each axis has a direction and length, with the longest axis occupying the approximated axonal direction. Four commonly used DTI measures for quantifying white matter integrity are axial diffusivity, radial diffusivity, mean diffusivity, and fractional anisotropy (FA). The first three diffusivity measures quantify diffusivity along the axonal direction, mean diffusivity perpendicular to the axonal direction, and overall mean diffusivity regardless of axonal direction, respectively. FA is approximately the differences in lengths among the three axes proportional to the overall diffusivity. FA is close to 1 if there is high anisotropy of water diffusion (presumably due to intact axons/myelin) at the voxel and close to 0 if there is low anisotropy.

WM integrity has been found to be a major predictor of cognition from childhood to old age such that more intact WM integrity is associated with better task performance (Bendlin et al., 2010; Kantarci et al., 2011). More recent studies related integrity of specific WM tracts to cognition. Using tractography to derive WM tracts in a group of participants ranging from 18 to 85 years old, Voineskos et al. (2012) identified positive relationships between the integrity of specific WM tracts and performance on cognitive domains derived from structural equation modeling (SEM). In a model that included age, FA for three WM tracts, and performance in three cognitive domains, the authors reported that integrity of the inferior longitudinal fasciculus (ILF) was associated with visuomotor dexterity, integrity of the occipitofrontal fasciculus was associated with visuospatial construction, and integrity of the splenium of the corpus callosum was associated with memory and executive functions. Voxel-wise analysis of skeletonised WM integrity maps also showed positive correlations between integrity of major WM tracts and general fluid intelligence (abstract reasoning and problem solving) in a study conducted by Haasz et al. (2013) involving a sample of middle-aged and elderly adults. Thus, better WM integrity of specific WM tracts has been associated with specific cognitive abilities.

Similar to other neuroimaging modalities, integrity measures for WM tracts are highly collinear (Penke et al., 2010; Lovden et al., 2013) and extremely noisy (Jaermann et al., 2004). Multivariate analysis takes advantage of this collinearity, allowing more sensitive extraction of signals from noisy data. This makes it an ideal tool for neuroimaging analyses (Habeck et al., 2010). Habeck et al. (2010) showed that when the effects of interest share common variances, multivariate analysis is more robust against noisy data than univariate techniques. Two commonly used multivariate techniques are factor analysis using principal component analysis (PCA) and structural equation modeling (SEM). Factor analysis allows quantifying the major sources of variance in the WM tracts across all subjects while SEM is used to partition the variances among factors. Penke et al. (2010) related the first principal component (PC), obtained from factor analysis on the fractional anisotropy (FA) for eight WM tracts, to a speed factor. This first PC accounted for $40.3 \%$ of the variance in FA and was negatively correlated with speed. In Johnson et al. (2015), the authors used the first two PCs for FA and related the scores to age and perceptual speed. Lovden et al. (2013) used SEM to compare a model positing a general whole brain factor for WM integrity across eight tracts and a model allowing tract-specific factors. The authors reported better fit indices for the tract-specific model despite high loadings for all tracts in the general factor model. These studies showed the utility of multivariate techniques in DTI data.

Building on these studies, our study applied Scaled Subprofile Modeling (SSM) analysis to our data, which is a well-established covariance technique that linearly combines the most meaningful PCs to predict an outcome variable (Habeck et al., 2008). This technique has been successfully applied to a number of imaging modalities such as arterial spin labeling (ASL) (Asllani et al., 2008), fludeoxyglucose positron emission tomography (FDG-PET) (Habeck et al., 2008), and Pittsburgh compound B (PIB-PET) (Oh et al., 2014), all of which consist of highly collinear data points. We extracted the mean FA of 18 major WM tracts using TRACULA (Tracts Constrained by Underlying Anatomy) (Yendiki et al., 2011), which displayed high collinearity among themselves. These tracts connect the major cortical regions and may constitute discrete networks for processing distinct cognitive abilities. We used SSM to identify a covariance pattern of WM tracts whose expression predicted a specific cognitive ability. By examining the loadings for the different WM tracts in each pattern we could identify the tracts that are most influential to each ability. Since age was not used in the extraction of these WM covariance patterns, we were then able to test for the ageindependent effect of WM on cognition and the indirect effect of age on cognition via WM.

\section{Method}

\section{Participants}

Three hundred forty six participants were included in the current analyses, 244 of which were from the Reference Ability Neural Network (RANN) study and 102 were from the Cognitive Reserve study. fMRI data from 174 of the RANN participants were included in Stern et al. (2014) but only the DTI data and neuropsychological test scores for these participants were used in the current study. Thus, the data used in the current analyses have not been published yet. Table 1 shows details of the participants' demographic information. Participants were recruited using established market mailing procedures to equate the recruitment procedures of young and old adults. Participants who responded to the mailing were telephone screened to ensure that they met basic inclusion criteria (right handed, English speaking, no psychiatric or neurological disorders that can affect cognition, normal or corrected-to-normal vision, and not taking any CNS-targeting medications). Individuals that passed the telephone screen were further screened in person and a Mattis Dementia Rating Scale score of at least 130 was required for inclusion in the study. Informed consent, as approved by the Internal Review Board of the College of Physicians and Surgeons of Columbia University, was obtained prior to study participation, and after the nature and risks of the study were explained. Participants were paid for their participation in the study.

\section{Cognitive tasks}

Four domains of cognitive functioning were assessed with three tasks each. A summary score was calculated for each domain by averaging the $\mathrm{z}$-scores of the three tasks under each domain.

\section{Memory}

Three sub-scores of the Selective Reminding Task (SRT) (Buschke and Fuld, 1974) were selected. Participants in this task were initially read a list of 12 words and asked to recall as many as they could. For the following five trials they were reminded of the words that they did not report and were asked to again recall all of the words in the list. Words are considered to enter long term storage from the point when they are recalled twice in a row without reminders. The longterm storage sub-score (SRTLTS) is the sum over all words of the number of trials when each word was in long-term storage. Continuous long-term retrieval (SRT CLRT) is the sum over all words of the number of trials for which the word was continuously recalled. The third memory measure was the number of words recalled on the last trial (SRT Last).

\section{Perceptual speed}

One selected measure was the score on the Digit Symbol subtest from the Wechsler Adult Intelligence Scale (WAIS III) (Wechsler, 1997). Participants in this test were instructed to write the symbol corresponding to specific numbers as quickly as possible based on a key specifying the appropriate symbol for each digit. The score is the number of correctly produced symbols in $90 \mathrm{~s}$. A second measure was the score on Part A of the Trail Making Test (Reitan, 1958), in which 
Table 1

Demographic information for 346 participants

\begin{tabular}{|c|c|c|c|c|c|c|}
\hline Decades & $20 s$ & $30 \mathrm{~s}$ & $40 \mathrm{~s}$ & $50 \mathrm{~s}$ & $60 \mathrm{~s}$ & $70 \mathrm{~s}$ \\
\hline$n$ & 86 & 47 & 33 & 41 & 99 & 40 \\
\hline Sex & $31 \mathrm{M} / 55 \mathrm{~F}$ & $15 \mathrm{M} / 32 \mathrm{~F}$ & $19 \mathrm{M} / 14 \mathrm{~F}$ & $20 \mathrm{M} / 21 \mathrm{~F}$ & $47 \mathrm{M} / 52 \mathrm{~F}$ & $21 \mathrm{M} / 19 \mathrm{~F}$ \\
\hline Age mean (SE) & $25.4(.278)$ & $34.1(.443)$ & $44.6(.458)$ & $53.7(.454)$ & $64.7(.258)$ & $73.5(.405)$ \\
\hline DRS mean $(S E)^{\mathrm{a}}$ & $140(.267)$ & $140(.382)$ & $140(.456)$ & $140(.484)$ & $140(.256)$ & $139(.472)$ \\
\hline
\end{tabular}

a DRS scores are not significantly different across decades.

participants are instructed to connect circles numbered from 1 to 24 as rapidly as possible and performance is assessed as the time to connect all 24 circles. The third speed measure was the number of colored ink patches named in $45 \mathrm{~s}$ in the Stroop Color Naming test.

\section{Reasoning ability}

One test was the WAIS III (Wechsler, 1997) Block Design test, in which participants are asked to reproduce a series of increasingly complex geometrical shapes using 4 or 9 identical blocks with red, white, or split red and white sides. A second test was the WAIS III (Wechsler, 1997) Letter-Number Sequencing test in which participants are asked to recall progressively longer lists of intermixed letters and numbers in alphabetical and then numerical order. The third reasoning test was the Raven's Progressive Matrices test (Raven, 1962) in which participants are asked to select which pattern in a set of eight possible patterns best completes a missing cell in a matrix.

\section{Vocabulary}

Tasks included the Vocabulary subtest from the WAIS III (Wechsler, 1997), the Wechsler Test of Adult Reading (WTAR) (Wechsler, 1997), and the American National Adult Reading Test (AMNART) (Grober and Sliwinski, 1991). The Vocabulary subtest asks participants to provide definitions for a series of increasingly advanced words, and the WTAR and AMNART both involve participants correctly pronouncing irregularly spelled English words.

\section{MRI acquisition}

MRI images were acquired in a 3.0 T Philips Achieva Magnet using a standard quadrature head coil. A T1-weighted scout image was acquired to determine subject position. One hundred sixty five contiguous $1 \mathrm{~mm}$ coronal T1-weighted images of the whole brain were acquired for each subject with an MPRAGE sequence using the following parameters: TR $6.5 \mathrm{~ms}$, TE $3 \mathrm{~ms}$; flip angle $8^{\circ}$, acquisition matrix $256 \times 256$ and $240 \mathrm{~mm}$ field of view. The DTI images were acquired in 55 directions using these parameters: $\mathrm{b}=800 \mathrm{~s} / \mathrm{mm}^{2}, \mathrm{TE}=69 \mathrm{~ms}$, TR $=$ $11,032 \mathrm{~ms}$, flip angle $=90^{\circ}$, in-plane resolution $112 \times 112$ voxels, acquisition time $12 \mathrm{~min} 56 \mathrm{~s}$, slice thickness $=2 \mathrm{~mm}$ (no gap), and 75 slices.

Any T1 scans with potentially clinically significant findings, such as abnormal neural structure, were reviewed by a neuroradiologist and removed from the sample prior to the current analysis. However, no clinically significant findings were identified or removed.

\section{Structural T1 processing}

T1 segmentation was a necessary step in processing the DTI data. Each subject's structural T1 scans were reconstructed using FreeSurfer v5.1 (http://surfer.nmr.mgh.harvard.edu/). The accuracy of FreeSurfer's subcortical segmentation and cortical parcellation (Fischl et al., 2002, 2004) has been reported to be comparable to manual labeling. Each subject's white and gray matter boundaries as well as gray matter and cerebral spinal fluid boundaries were visually inspected slice by slice, manual control points were added in the case of any visible discrepancy, and reconstruction was repeated until we reached satisfactory results within every subject. The subcortical structure borders were plotted by FreeView visualization tools and compared against the actual brain regions. In case of discrepancy, they were corrected manually.

DTI analysis

DTI data were processed with TRACULA (Tracts Constrained by Underlying Anatomy) distributed as part of the FreeSurfer v. 5.2 library (Yendiki et al., 2011) which produces 18 major WM tracts as listed below:

- bilateral corticospinal tract (CST)

- bilateral inferior longitudinal fasciculus (ILF)

- bilateral uncinate fasciculus (UNC)

- bilateral anterior thalamic radiation (ATR)

- bilateral cingulum-cingulate gyrus (supracallosal) bundle (CCG)

- bilateral cingulum-angular (infracallosal) bundle (CAB)

- bilateral superior longitudinal fasciculus-parietal bundle (SLFP)

- bilateral Superior longitudinal fasciculus-temporal bundle (SLFT)

- forceps major, which passes through genu of corpus callosum (FMAJ)

- Forceps minor, which passes through splenium of corpus callosum (FMIN).

The software performs informed automatic tractography by incorporating anatomical information from a training data set, provided by the software, with the anatomical segmentation of the T1 image of the current data set, thus increasing the accuracy of the WM tract placement for each participant. Standard DTI processing steps using the FMRIB's Diffusion Toolbox (FMRIB's Software Library v. 4.1.5) including eddy current correction, tensor estimation, and bedpostx were performed prior to tractography. See Yendiki et al. (2011) for detailed steps performed by the TRACULA software. For each participant, the means of fractional anisotropy (FA) for each of the 18 tracts, were entered into subsequent analyses. FA ranges from 0 to 1 with higher number representing more intact WM integrity.

\section{Statistical analyses}

\section{Cognitive abilities}

z-Scores were calculated for each cognitive task among all decades. For each cognitive ability, a summary score was then calculated by averaging the z-scores of the three tasks for each ability. Thus each participant had four summary scores, one for each cognitive ability such that higher scores indicated better performance.

\section{WM integrity covariance patterns}

For each cognitive ability, we used the Scaled Subprofile Modeling (SSM) approach (Habeck et al., 2008) to derive a covariance pattern, consisting of a set of PCs, that best predicted performance in each ability across all participants. The steps of SSM are as follows:

(1) Performed PCA on all WM tract data across all subjects.

(2) In order to determine the optimal linear combination of PCs whose expression best predicts each cognitive ability, brain behavior regression was performed in which the independent variables were the subject loadings from PCs, and the dependent variable was the performance measure. AIC was then employed 
to select the best-fitting subset of all possible 18 subsets, i.e., PC1, PC1 and PC2, PC1 and PC2 and PC3, and etc.

(3) Pattern construction: After the optimal subset is selected, we took the regression weights for all of the chosen components and constructed a corresponding pattern which assigned a loading to each tract.

(4) Bootstrap procedure: So far, we had only produced a point estimate of the best-fitting tract pattern, we had no information yet about the robustness of the individual tract loadings. With the bootstrap procedure, we sampled from the data with replacement, and repeated PCA, brain-behavior regression with the subset selected in step 2, and pattern construction 10,000 times. AIC was just employed for the purpose of subset selection and was only done once in step 2 . After 10,000 iterations, we computed the variability of tract loadings around the point estimate value and produced $95 \%$ confidence intervals for the pattern loading of each WM tracts, which provided information on the stability of the contribution from each WM tract towards the covariance pattern. Confidence intervals that did not overlap with zero demonstrated a tract's significant contribution towards the covariance pattern.

In this manner, a unique covariance pattern was derived for each cognitive ability for which the pattern expression across subjects optimally predicted the corresponding ability. Pattern expression scores for each covariance pattern were calculated as described above and were used in subsequent analyses.

\section{Effects of age and WM covariance pattern expression on cognition}

Separate regression models were conducted for each cognitive ability with age, WM covariance pattern expression, and their interaction as factors and the cognitive ability as the dependent variable. Analyses were performed in SPSS (v22; IBM) and the alpha level for all analyses was set at 0.05 .

\section{Mediation analysis}

Mediation was performed for each cognitive ability to examine whether the WM integrity patterns explained some of the age-related differences in cognition. Even though each pattern was derived based on best prediction for each ability, age was never used to derive the patterns, thus avoiding circularity when testing for mediation of agerelated differences in cognition. Mediation analyses were performed with PROCESS, a macro for SPSS (Hayes, 2013), with 5000 stratified bootstrap resamples to determine the bias-corrected percentile confidence intervals for the indirect effect of WM integrity. Confidence intervals that did not overlap with zero provided evidence of significant indirect effect for the mediator.

\section{Results}

\section{Correlations among age, cognition, and WM integrity}

As shown in previous studies (Salthouse and Ferrer-Caja, 2003; Lindenberger, 2014), with increasing age, performance on the summary measures of speed, memory, and reasoning decreased (speed: $\mathrm{r}=-5.71$; memory: $\mathrm{r}=-.465$; reason: $\mathrm{r}=-.385$; all $\mathrm{p}<.05$ ) while vocabulary increased $(\mathrm{r}=.245, \mathrm{p}<.05)$. Fig. 1 shows the mean z-scores for each cognitive ability in each decade. $z$-Scores were computed within each cognitive task with all age groups combined. Table 2 shows the Pearson correlation coefficients for the associations among age, each of the 4 cognitive abilities, and the mean FA for the 18 WM tracts. Correlations among mean FAs and each of the cognitive abilities were controlled for the effects of the other three abilities to show correlations unique to each ability. The correlations were corrected for multiple comparisons across 18 tracts $(\mathrm{p}<0.05 / 18=$ .0027). With the exception of bilateral CAB, the right SLFT, and the right SLFP, all other WM tracts were negatively correlated with age. Five WM tracts were positively correlated with Speed after controlling for the other three abilities. Only forceps minor was positively correlated with Reasoning, and none with Memory. Vocabulary was negatively correlated with forceps minor and left CST.

\section{WM covariance patterns}

Using SSM, a separate covariance pattern was identified whose expression predicted cognitive performance in each ability. Fig. 2 shows the number of PCs selected based on the lowest AIC and were linearly combined to create the covariance patterns for each of the three

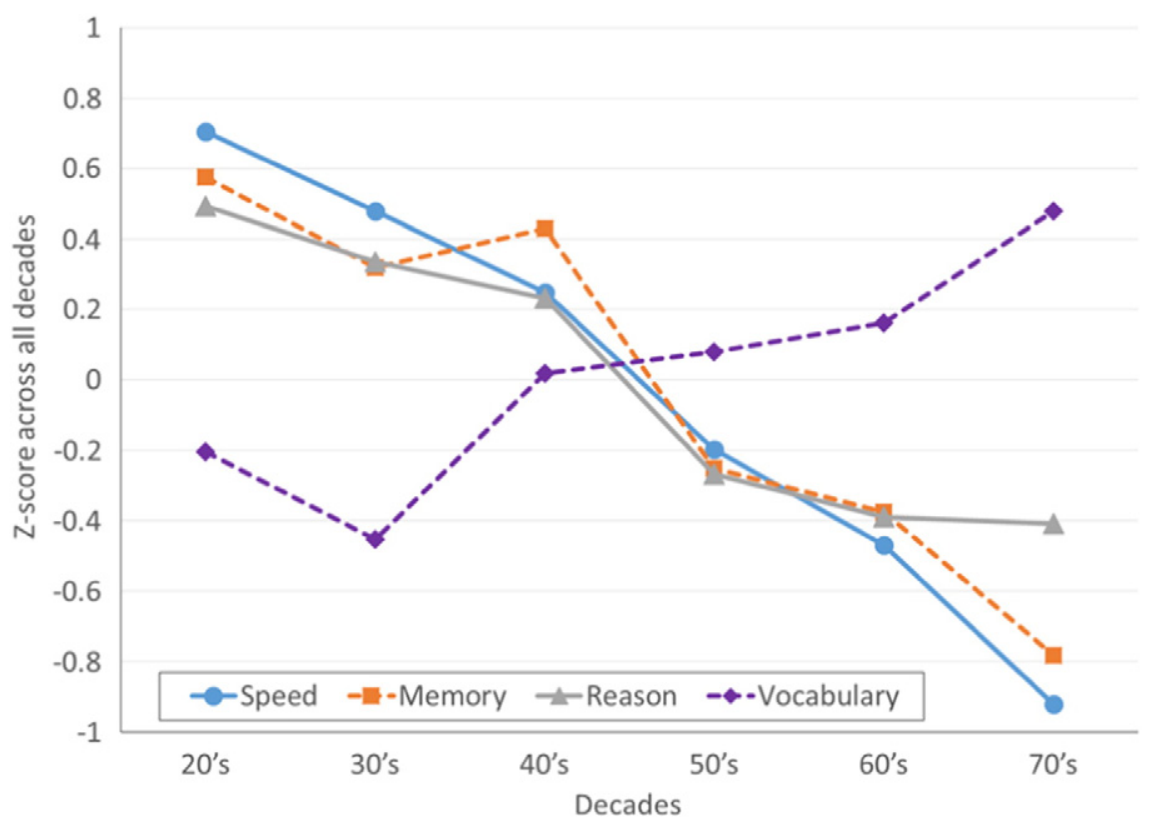

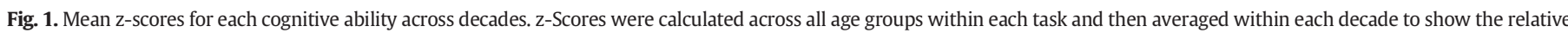
trend in performance across age groups. Solid and dashed lines helped to distinguish the overlapping lines from each other. 
Table 2

Correlation among age, cognitive abilities, and FA for $18 \mathrm{WM}$ tracts. Correlations with each cognitive ability were partialled for the other three abilities.

\begin{tabular}{|c|c|c|c|c|c|}
\hline WM tracts & Age & Speed & Memory & Reasoning & Vocabulary \\
\hline fmajor & $-.176^{* *}$ & $0.139^{* *}$ & 0.066 & 0.003 & -0.051 \\
\hline fminor & $-.446^{* *}$ & 0.086 & 0.057 & $0.228^{* *}$ & $-0.308^{* *}$ \\
\hline L ATR & $-.248^{* *}$ & $0.184^{* *}$ & -0.003 & 0.065 & -0.057 \\
\hline L CAB & $.132 *$ & -.072 & 0.018 & 0.032 & 0.019 \\
\hline L CCG & $-.214^{* *}$ & $0.159^{* *}$ & -0.026 & 0.09 & -0.039 \\
\hline L CST & $-.328^{* *}$ & 0.081 & 0.088 & $0.119^{*}$ & $-0.233^{* *}$ \\
\hline L ILF & $-.340^{* *}$ & $0.168^{* *}$ & $0.117^{*}$ & 0.064 & -0.088 \\
\hline L SLFP & $-.311^{* *}$ & $0.160^{* *}$ & 0.024 & 0.071 & $-0.113^{*}$ \\
\hline L SLFT & $-.232^{* *}$ & $0.168^{* *}$ & -0.004 & 0.054 & -0.019 \\
\hline L UNC & $-.243^{* *}$ & $0.125^{*}$ & 0.066 & 0.082 & -0.042 \\
\hline R ATR & $-.242^{* *}$ & $0.116^{*}$ & -0.018 & $0.130^{*}$ & $-0.156^{* *}$ \\
\hline R CAB & -.028 & 0.061 & -0.004 & 0.005 & -0.001 \\
\hline R CCG & $-.224^{* *}$ & $0.185^{* *}$ & -0.018 & 0.028 & $-0.130^{*}$ \\
\hline R CST & $-.209^{* *}$ & 0.068 & 0.002 & $0.152^{* *}$ & $-0.115^{*}$ \\
\hline R ILF & $-.382^{* *}$ & $0.185^{* *}$ & 0.096 & 0.067 & -0.099 \\
\hline R SLFP & $-.117^{*}$ & 0.103 & -0.036 & 0.060 & -0.007 \\
\hline R SLFT & -.080 & 0.099 & -0.048 & 0.038 & -0.001 \\
\hline R UNC & $-.295^{* *}$ & $0.134^{*}$ & 0.093 & 0.090 & -0.092 \\
\hline
\end{tabular}

${ }^{*} \mathrm{p}<.05 ;{ }^{* *} \mathrm{p}<.01$, bolded text: $\mathrm{p}<.002$ corrected for multiple comparison.

abilities. The subject expression scores of the patterns significantly predicted each cognitive ability when controlling for age with the exception of vocabulary (statistical details are provided in Table 3 and Fig. 2). In Fig. 2B, blue dots represent the point estimates for the loadings of each WM tract in the covariance pattern (= linearly combined loadings for the set of selected PCs) and the red bars are the bootstrapped $95 \%$ confidence intervals for each WM tract in the covariance pattern associated with each cognitive ability. WM tracts with loadings of the highest absolute values are the most influential in the patterns, and confidence intervals that do not overlap with zero indicate significant contribution to the covariance pattern. Only two WM tracts showed nonzero influence on the covariance pattern for memory, six WM tracts for Speed, and twelve tracts for reasoning. Please see Fig. 2 for the identities of the specific tracts.
Table 3

F-values for the effects of Age and WM pattern expression.

\begin{tabular}{llccc}
\hline & Speed & Memory & Reason & Vocabulary \\
\hline Age & $84.7^{* * *}$ & $50.0^{* * *}$ & $29.5^{* * *}$ & $13.0^{* * * *}$ \\
Pattern expression & $13.3^{* * *}$ & $5.16^{*}$ & $6.24^{*}$ & 2.49 \\
\hline
\end{tabular}

Note. Dfs $=1,343$.

${ }^{*} \mathrm{p}<.05$.

*** $\mathrm{p}<.001$.

Interaction of age by pattern expression on cognitive abilities

In order to ensure that the association between pattern expression and each of the cognitive abilities did not differ by age, the interaction between age and pattern expression was tested for each ability. The interaction effect was not significant for each of the four abilities: speed: $\mathrm{F}(1,342)=0.403$; reason: $\mathrm{F}(1,342)=0.262$; memory: $\mathrm{F}(1,342)=$ 0.001 ; vocabulary: $F(1,342)=0.166$, all $\mathrm{p}>.50$. Thus, the pattern expression to cognitive ability relationship did not change as a function of age. This observation allowed us to test the independent effects of age and pattern expression on cognition and to proceed with the simple mediation analysis, as described below.

\section{Age-independent effect of WM pattern expression}

We also evaluated the effects of age and pattern expression on each cognitive ability. As summarized in Table 3, for speed, memory, and reasoning, their associated pattern expression significantly predicted each cognitive ability even after controlling for age. However, WM covariance pattern expression for vocabulary did not predict vocabulary after controlling for age.

\section{Indirect effect of age on cognition via WM pattern expression}

Having established that pattern expression significantly predicts cognitive ability and that the associations do not differ with age, mediation of WM covariance patterns on age-related differences in cognition was tested for each ability. As shown in Fig. 1A, increasing age was

A
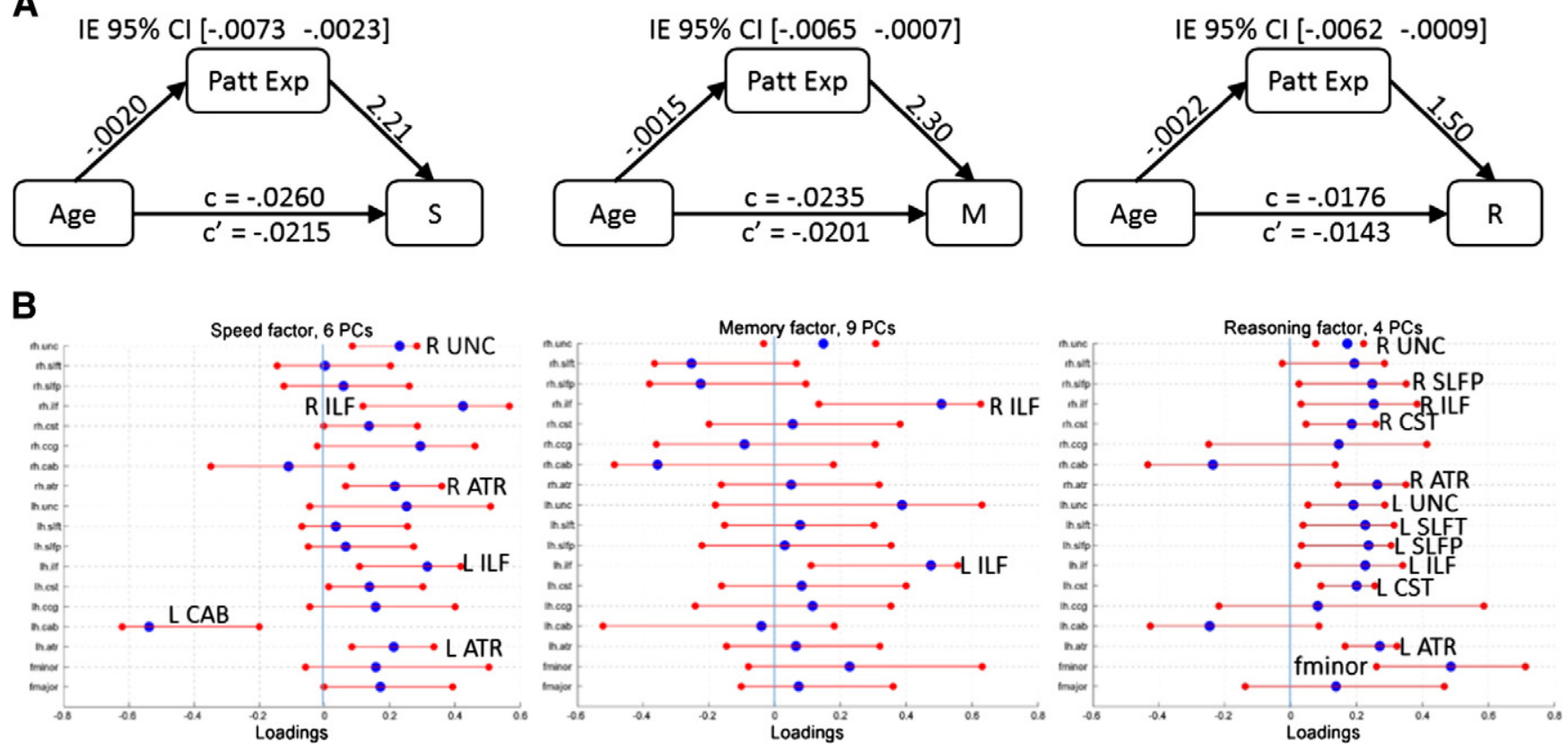

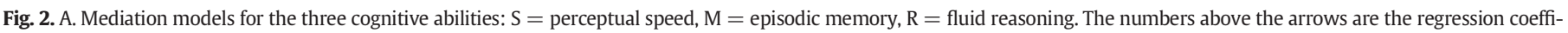

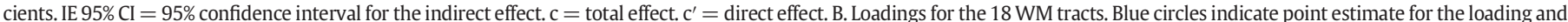

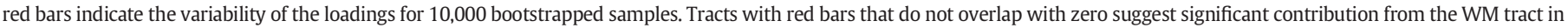

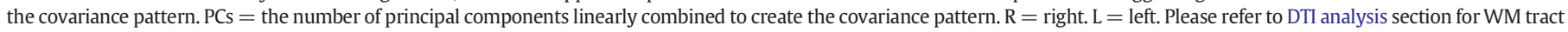
abbreviations. 
associated with lower pattern expression, and higher pattern expression was associated with better cognitive ability for speed, memory, and reasoning. The bracketed numbers above each mediation diagram indicates the confidence interval for the indirect effect of the WM covariance pattern. All of the confidence intervals do not overlap with zero, demonstrating that the indirect effect for WM covariance patterns on all three cognitive abilities are significant.

\section{Discussion}

The aim of the study was to examine the relationships among age, FA of 18 major WM tracts, and four cognitive abilities (perceptual speed, episodic memory, fluid reasoning, and vocabulary) by investigating if (1) FA exerts an age-independent effect on cognition, and (2) if FA indirectly explains some of the age-related differences in the four cognitive abilities. We summarized the FA for 18 WM tracts by extracting a covariance pattern of WM tracts for each cognitive ability, and then tested the relationships between the expression of each WM covariance pattern with age and the corresponding cognitive ability. After confirming that the interaction between age and WM covariance pattern expression was not significant for all four cognitive abilities, we proceeded to testing for the age-independent effect of WM pattern on each cognitive ability. While all of the cognitive abilities were significantly affected by age, with the exception of vocabulary, WM covariance patterns had significant effects on each of the three cognitive abilities even after controlling for age. Thus, for the three abilities that decline with age, WM covariance patterns do exhibit an age-independent effect on cognition.

Furthermore, an indirect effect of age on cognition was found via expressions of the WM covariance patterns for three of the four abilities, with vocabulary also being the exception. This demonstrates that a portion of the age-related differences in cognition are attributable to differences in WM integrity for abilities that decrease with age. Hedden et al. (2014) found that mean whole brain WM integrity, among other brain measures, was one of the strongest mediators of the age-cognition association. Our study further advances our understanding of the age-WM integrity-cognition relationships by localizing the influence of WM integrity to specific major WM tracts for each cognitive ability.

The WM covariance patterns derived to predict performance on each ability consisted of a linear combination of principal components (PCs) that accounted for the major sources of variance in FA across all 18 WM tracts. While previous studies usually incorporated at most the first two PCs of a principal component analysis (PCA) into their analyses, the current study demonstrated that greater specificity can be achieved by incorporating more PCs into the WM covariance pattern. While these patterns were specifically extracted to predict each of the abilities, age was not included in the derivation and therefore does not guarantee mediation of the age-related effect on cognitive abilities. This was clearly shown in the lack of significant mediation by the WM covariance pattern derived for the vocabulary ability. Thus, mediation by the three WM covariance patterns derived for perceptual speed, episodic memory, and fluid reasoning represent statistically significant mediation effects rather than a product of the pattern derivation process.

\section{WM tracts associated with each cognitive ability}

Each of the WM covariance patterns associated with cognitive abilities showed major influences from the FA of a set of WM tracts. The WM covariance pattern derived for perceptual speed showed the largest indirect effect for age-related differences and involved a moderate number of tracts that are most influential in the pattern. These tracts consisted of positive contributions from bilateral anterior thalamic radiation (ATR) and inferior longitudinal fasciculus (ILF), and right uncinate fasciculus, and negative contributions from the left parahippocampal cingulum ( $\mathrm{CAB}$ ). Involvement of these positively weighted tracts in the speed pattern is consistent with the nature of the tasks. The speed ability was composed of scores from the Digit Symbol, Trails A, and color naming in the Stroop task, all of which require integration of sensory information from the thalamus to the frontal cortex through the ATR and also from temporal and occipital cortices through the ILF. While Penke et al. (2010) reported a general WM integrity factor predicting speed ability, our results point to more specific tracts that are most influential in the speed ability. Negative loading from the left $\mathrm{CAB}$ suggest that high integrity is associated with worse speed ability, which has been reported in previous studies (Hoeft et al., 2007) and may be due to overproduction of myelin on the axonal tracts and/or changes in the axon such as decreased axonal diameter or packing density (Beaulieu, 2002).

The WM covariance pattern associated with the fluid reasoning ability consisted of contributions from the most number of WM tracts, which is consistent with our understanding of the reasoning ability. Reasoning tasks place demands on many more processes than the other three abilities do, including processes such as visuospatial integration, logical reasoning, visuospatial reasoning, and working memory. Although a voxel-wise approach was used, Haasz et al. (2013) also reported significant correlation between FA and fluid reasoning in a substantial portion of the voxels in the major WM tracts including forceps minor, left superior and inferior longitudinal fasciculus, bilateral inferior fronto-occipital fasciculus, left uncinate fasciculus, left cingulum, and the right anterior thalamic radiation, most of which showed high loadings in our pattern as well.

For the memory WM covariance pattern, one would expect connections between the frontal and the temporal lobes, but only bilateral ILF, which connects the temporal and occipital lobes, showed significant contribution to the covariance pattern. This is not surprising because in a study that performed tractography for major WM tracts, the ILF shares $>10 \%$ spatial overlap with the inferior fronto-occipital fasciculus (IFO), which connects frontal and occipital lobes, and the correlation between ipsilateral ILF and IFO is .77 and .66 for the left and right hemisphere, respectively (Wahl et al., 2010). However, TRACULA does not perform tractography for IFO as of now. Thus, the association observed between ILF and memory can most likely be attributed to the similarity shared by ILF and IFO.

\section{Vocabulary ability}

In the current result as well as in previous studies, vocabulary ability has been found to be maintained rather than decline with aging (Salthouse and Ferrer-Caja, 2003). Even though a pattern was derived that predicted vocabulary, the effect was no longer significant once age was accounted for. A direct interpretation of the result is that other brain measures exert greater influence on the vocabulary ability than WM integrity such as gray matter volume and thickness and functional activation. Another possibility is that the vocabulary ability does not rely on the distributed cooperation of major long distance WM tracts. Habeck et al. (2010) showed that when the effects of interest are more focal, i.e., affecting only regions that share minimal variance with each other, then multivariate analysis may not be able to extract the effect of interest as well as a univariate approach would. Vocabulary processes likely rely more on specific WM connections lateralized to the left hemisphere (all participants were right dominant) and rely on tracts limited to the inferior frontal to temporal connections. Since the 18 WM tracts examined in the current study was not specific to the vocabulary ability, future studies should use vocabulary-related brain regions as seeds for tractography analysis to extract the WM tracts most relevant to the vocabulary ability to examine the association between WM health and the vocabulary ability.

\section{White matter integrity measures}

In our analysis, we only performed SSM on the FA measure even though most studies commonly report results for at least two of the 
four white matter integrity measures. This is a conscious choice based on the fact that factor analyses on all four measures for each WM tract (total of 18 factor analyses) showed that the first factor accounts for 58 to $76 \%$ of the variance across subjects (data not reported here). This can be attributed to the fact that the four measures are just different ways to combine the same three numbers - the lengths of the three vectors describing the ellipsoid. If we test the same hypothesis on all four measures, we would be performing multiple comparisons and increasing the type I error rate by 4 fold, but if we correct for multiple comparisons with Bonferroni correction, for example, then we would be imposing an overly stringent correction given that the measures are highly correlated and increasing type II error rate. If the same SSM analysis was performed on the other three diffusivity measures, the resulting covariance patterns should be similar to those reported here given the high degree of similarity among the measures. Future work should formally characterize the relationships among the four measures with Monte Carlo simulations, for example, and propose more statistically responsible approaches in working with the four white matter integrity measures.

\section{Caveat on mediation results}

While mediation techniques test hypothesized causal effects, when the data is cross-sectional as in the current study, caution is warranted when making causal claims. Generally, the major concern in the use of cross-sectional data to make causal inference is misrepresentation in the direction of the causal relationships. In the current study, when the independent variable (IV) is age, the mediator (M) is a brain measure, and the dependent variable (DV) is cognition, the direction of causality is less ambiguous than in most cases. Age has to be the IV because nothing else can influence chronological age. For the mediator and DV, the brain measure is most likely exerting effects on cognition rather than cognition influencing the brain measure based on the assumption that cognition is an outcome of the underlying structure. Cognitive intervention studies that show structural changes associated with cognitive changes seem to support the idea that cognitive changes can result in structural changes (Zatorre et al., 2012). Still it is more logical to assume that repeated stimulation of the associated neural circuit during cognitive training results in structural changes that are associated with improved cognition rather than positing that cognitive performance improves on its own which then causes structural changes. However, with just cross-sectional data, it is not possible to provide substantial proof of causal directions. Therefore, the use of crosssectional data in the current study is unlikely but still possible to have misinformed the direction of causality.

Another concern in making causal inferences was outlined in Maxwell and Cole (2007) in which the authors demonstrated that for true longitudinal models with time delays in the relationships between IV and mediator and mediator and DV, cross-sectional analysis might give biased estimates of indirect effects. However, in the current paper the true longitudinal relationships are not known yet; mediation was used to explore the structure of the associations among age, WM integrity, and cognition. Further, the statistical test performed in the current study has tight control on the type-I error (Hayes, 2013) such that no indirect effect would be found if age was merely affecting WM integrity and cognition independently, with no causal interaction of the latter two quantities. Thus, an indirect effect, while possibly biased with regard to possible longitudinal models, is unlikely to have arisen by chance.

Overall, while the causal relationships reported in the current study must be confirmed with longitudinal data, the finding of indirect effects between age and cognition via WM integrity are likely to be valid despite the use of cross-sectional data. An indirect effect through the WM covariance pattern occurs when there is a statistically robust decrease in the effect of age on each of the three cognitive abilities when taking into account expression of the WM covariance pattern. A direct interpretation of the indirect effect is that differences in the expression of the WM covariance pattern partially explains the effect of age on each of the cognitive abilities, potentially due to the aging mechanism acting on changes in white matter integrity which in turn results in cognitive decline. However, while the indirect effect is present in our data, interpretation of the indirect effect requires confirmation with longitudinal data.

\section{Conclusion}

Our study presented a comprehensive examination of the associations among age, FA of 18 major WM tracts, and 4 cognitive abilities across adulthood. Using a multivariate approach, we identified a WM covariance pattern that (1) shows age-independent effect on cognition, and (2) mediates the effect of age on cognition for perceptual speed, episodic memory, and fluid reasoning but not for vocabulary. The covariance patterns consisted of major influences from specific sets of WM tracts that were different across the three abilities. Our results suggest that for cognitive abilities that decline with age, the integrity of WM tracts are associated with each ability over and above age effects and that the aging effect on cognitive decline may exert its influence through WM integrity changes.

\section{Acknowledgments}

This work was supported by NIH/NIA R01 AG26158, NIH/NIA R01 AG038465, and NIH UL1 TR000040/NCRR UL1 RR024156.

\section{References}

Asllani, I., et al., 2008. Multivariate and univariate analysis of continuous arterial spin labeling perfusion MRI in Alzheimer's disease. J. Cereb. Blood Flow Metab. 28 (4), 725-736.

Beaulieu, C. 2002. The basis of anisotropic water diffusion in the nervous system - a technical review. NMR Biomed. 15 (7-8), 435-455.

Bendlin, B.B., et al., 2010. White matter in aging and cognition: a cross-sectional study of microstructure in adults aged eighteen to eighty-three. Dev. Neuropsychol. 35 (3), 257-277.

Buschke, H., Fuld, P.A., 1974. Evaluating storage, retention, and retrieval in disordered memory and learning. Neurology 24 (11), 1019-1025.

Fischl, B., et al., 2002. Whole brain segmentation: automated labeling of neuroanatomical structures in the human brain. Neuron 33 (3), 341-355.

Fischl, B., et al., 2004. Automatically parcellating the human cerebral cortex. Cereb. Cortex 14 (1), 11-22.

Grober, E., Sliwinski, M., 1991. Development and validation of a model for estimating premorbid verbal intelligence in the elderly. J. Clin. Exp. Neuropsychol. 13 (6), 933-949.

Haasz, J., et al., 2013. General fluid-type intelligence is related to indices of white matter structure in middle-aged and old adults. Neurolmage 83C, 372-383.

Habeck, C., et al., 2008. Multivariate and univariate neuroimaging biomarkers of Alzheimer's disease. NeuroImage 40 (4), 1503-1515.

Habeck, C., Stern, Y., Alzheimer's Disease, I., 2010. Neuroimaging, multivariate data analysis for neuroimaging data: overview and application to Alzheimer's disease. Cell Biochem. Biophys. 58 (2), 53-67.

Hayes, A.F., 2013. Introduction to mediation. Hayes, Moderation, and Conditional Process Analysis: A Regression-based ApproachMethodology in the Social Sciences. Guilford Publications, New York (pages cm).

Hedden, T., et al., 2014. Multiple brain markers are linked to age-related variation in cognition. Cereb. Cortex, http://dx.doi.org/10.1093/cercor/bhu238.

Hoeft, F., et al., 2007. More is not always better: increased fractional anisotropy of superior longitudinal fasciculus associated with poor visuospatial abilities in Williams syndrome. J. Neurosci. 27 (44), 11960-11965.

Jaermann, T., et al., 2004. SENSE-DTI at 3 T. Magn. Reson. Med. 51 (2), 230-236.

Johnson, M.A., Diaz, M.T., Madden, D.J., 2015. Global versus tract-specific components of cerebral white matter integrity: relation to adult age and perceptual-motor speed. Brain Struct. Funct. 220 (5), 2705-2720.

Kantarci, K., et al., 2011. Diffusion tensor imaging and cognitive function in older adults with no dementia. Neurology 77 (1), 26-34.

Lindenberger, U., 2014. Human cognitive aging: corriger la fortune? Science 346 (6209), 572-578.

Lovden, M., et al., 2013. The dimensionality of between-person differences in white matter microstructure in old age. Hum. Brain Mapp. 34 (6), 1386-1398.

Maxwell, S.E., Cole, D.A., 2007. Bias in cross-sectional analyses of longitudinal mediation. Psychol. Methods 12 (1), 23-44.

Moss, M.B., et al., 2007. Successful vs. unsuccessful aging in the rhesus monkey. In: Riddle, D.R. (Ed.), Brain Aging: Models, Methods, and Mechanisms. Boca Raton (FL). 
Oh, H., et al., 2014. Association of gray matter atrophy with age, beta-amyloid, and cognition in aging. Cereb. Cortex 24 (6), 1609-1618.

Penke, L., et al., 2010. A general factor of brain white matter integrity predicts information processing speed in healthy older people. J. Neurosci. 30 (22), 7569-7574.

Raven, J.C., 1962. Advanced Progressive Matrices, Set II. The Psychological Corporation, San Antonio, TX.

Reitan, R.M., 1958. Validity of the Trail Making test as an indicator of organic brain damage. Percept. Mot. Skills 8, 271-276.

Salthouse, T.A., 2005. Relations between cognitive abilities and measures of executive functioning. Neuropsychology 19 (4), 532-545.

Salthouse, T.A., Ferrer-Caja, E., 2003. What needs to be explained to account for agerelated effects on multiple cognitive variables? Psychol. Aging 18 (1), 91-110.

Salthouse, T.A., Pink, J.E., Tucker-Drob, E.M., 2008. Contextual analysis of fluid intelligence. Intelligence 36 (5), 464-486.

Stern, Y., et al., 2014. The reference ability neural network study: motivation, design, and initial feasibility analyses. NeuroImage 103, 139-151.
Voineskos, A.N., et al., 2012. Age-related decline in white matter tract integrity and cognitive performance: a DTI tractography and structural equation modeling study Neurobiol. Aging 33 (1), 21-34.

Wahl, M., et al., 2010. Microstructural correlations of white matter tracts in the human brain. Neurolmage 51 (2), 531-541.

Wechsler, D., 1997. Wechsler Adult Intelligence Scale - Third Edition. The Psychologica Corporation, San Antonio.

Yendiki, A., et al., 2011. Automated probabilistic reconstruction of white-matter pathways in health and disease using an atlas of the underlying anatomy. Front. Neuroinform. 5 23.

Zatorre, R.J., Fields, R.D., Johansen-Berg, H., 2012. Plasticity in gray and white: neuroimaging changes in brain structure during learning. Nat. Neurosci. 15 (4), 528-536. 